\title{
New design of multiple-cavity detector for high mass axion dark matter search
}

\author{
Junu Jeong*, Saebyeok Ahn \\ Department of physics, Korea Advanced Institute of Science and Technology \\ E-mail: jwpc0120@kaist.ac.kr, asb5229@kaist.ac.kr
}

\section{SungWoo Youn}

Center for axion and precision physics research, Institute for Basic Science

E-mail: swyoundibs.re.kr

\section{Jihn E. Kim}

Department of Physics, Kyung Hee University

Center for axion and precision physics research, Institute for Basic Science

E-mail: jihnekimegmail.com

\section{Yannis K. Semertzidis}

Department of physics, Korea Advanced Institute of Science and Technology Center for axion and precision physics research, Institute for Basic Science

E-mail: yannisekaist.ac.kr

\begin{abstract}
In cavity-based axion dark matter search experiments exploring high mass regions, arrays of multiple cavities are typically considered to increase the detection volume within a given magnet bore. We, IBS/CAPP at KAIST, introduce a new idea, referred to as pizza-cylinder cavity, which is superior to a conventional multiple-cavity detector in terms of detection volume, simplicity of the experimental setup, and facilitation of the phase-matching mechanism. This design is promising for detecting high frequency axion dark matter with enhanced experimental sensitivities. We present the characteristics of this concept and demonstrate the experimental feasibility using a double-cell cavity. (This work was supported by IBS-R017-D1-2018-a00 / IBS-R017-Y1-2018-
\end{abstract} $\mathrm{a} 00$.)

The 39th International Conference on High Energy Physics (ICHEP2018)

4-11 July 2018

Seoul, Republic of Korea

${ }^{*}$ Speaker. 
The axion is a hypothetical pseudoscalar particle, as a consequence of the PQ mechanism, to solve the strong CP problem in particle physics. If the mass of axion falls into a specific range, it can be a good candidate for cold dark matter. In a strong magnetic field, axions can be converted into photons with energy of the axion mass and resonated inside a cavity, known as haloscope.

As searching for higher mass axions requires a smaller cavity size, one can place several small cavities together inside a magnet bore to increase the detection volume. However, the volume usage is not efficient enough mainly due to unused volume and cavity thickness. Herein, we propose a new cavity concept, multiple-cell cavity (dubbed "pizza cavity"), that maximizes the volume usage for a given magnet as shown in Fig. 1. This design enhances the scanning speed substantially comparing to conventional multiple-cavity systems [1].
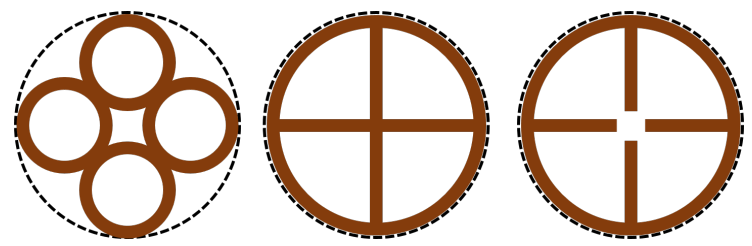

Figure 1: Cross-sectional view of various designs for multiple-detector system: multiple-cavity; multiple-cell cavity; multiple-cell cavity with a hollow gap in the middle. The dashed line represents the boundary of the magnet bore.

The hollow gap introduced in the middle of the cavity plays various critical roles. It breaks the mode degeneracy modes making the lowest mode be the $\mathrm{TM}_{010}$-like mode. It spatially connects all the cells enabling a single coupler to pick up signal from the entire volume, which simplifies the readout chain. It vanishes the electric fields of higher resonant modes at the center of the cavity once the overall electric field distributed symmetrically, referred to as phases-matching. Experimentally, the phase-matching is assured by vanishing of coupling strength for higher modes, which is visualized by disappearance of the corresponding reflection peaks (constant resistance circles) in the scattering parameter space (Smith chart) using a network analyzer.

Experimental demonstration for the new cavity concept is carried out at room temperature using a double-cell cavity. As seen in Fig. 2, the results show that the phase-matching requires less than 2 seconds to be accomplished, making a negligible effect to the DAQ efficiency. A good linearity in the resonant frequency over step indicates stability of the tuning mechanism. Based on the demonstration, we conclude this concept of multiple-detector system is promising for axion dark matter search exploring high frequency regions.
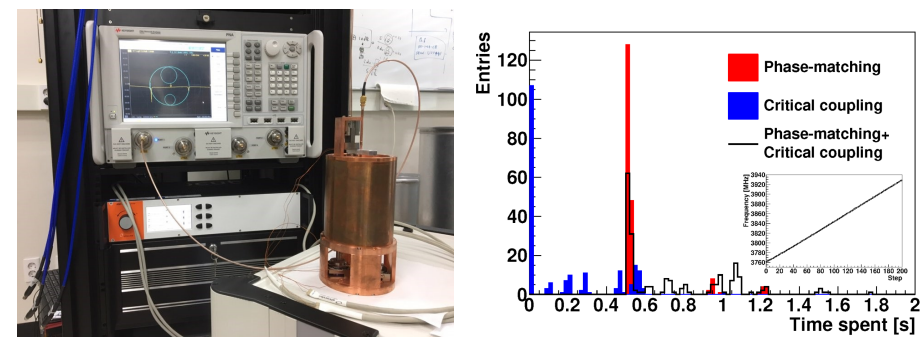

Figure 2: (Left) System setup for demonstration. (Right) Time durations for the tuning mechanism with the red and blue histograms representing the time required for phase-matching and critical coupling, respectively. The $\mathrm{TM}_{010}$-like resonant frequency with step is shown in the enclosed box.

\section{References}

[1] J. Jeong et al., Phys. Lett. B 777, 412 (2018). 